Check for updates

Cite this: RSC Adv., 2017, 7, 52261

Received 15th September 2017

Accepted 4th November 2017

DOI: $10.1039 / \mathrm{c} 7 \mathrm{ra10260 \textrm {g }}$

rsc.li/rsc-advances

\section{Direct growth of a graphitic nano-layer on optical fibers for ultra-fast laser application $\uparrow$}

\author{
Conghao Yang, $\$$ Ling Yun, + Yang Qiu, Hanqing Dai, (DD Detao Zhu, Zhijia Zhu, \\ Zuxing Zhang, * Kehan Yu (DD * and Wei Wei
}

Direct growth of graphitic nano-layer (GNL) on optical fiber is achieved using plasma-enhanced chemical vapor deposition. Clean and uniform GNL nanocrystalline flakes are grown in a controllable manner on the fiber-end without catalysts. $991 \mathrm{fs}$ soliton pulse generation at $1559 \mathrm{~nm}$ is achieved using the GNL coated optical fiber in an erbium-doped fiber laser.
Two-dimensional (2D) layered materials, including graphene, topological insulators, transition metal dichalcogenides, and black phosphorus, have been attracting increasing attention in photonic and optoelectronic applications. In particular, graphene is a $2 \mathrm{D}$ honeycomb lattice structure of carbon atoms characterized by a linear relation between energy and momentum, massless Dirac fermions, broad wavelength range and ultrafast relaxation mechanism, making it a promising saturable absorber (SA) for ultra-fast lasers. ${ }^{1-4}$ Several techniques have been developed for the fabrication of graphenebased saturable absorbers (GSAs), e.g. mechanical exfoliation, ${ }^{5,6}$ liquid phase exfoliation, ${ }^{7-9}$ chemical vapor deposition (CVD) ${ }^{10-13}$ graphene oxide (GO), ${ }^{9,14}$ and reduced GO. ${ }^{14,15}$ However, the existing methods of preparing GSAs usually suffer from complex procedure, residual polymer and metal catalysts, and mechanical damage. ${ }^{16}$ In addition, many reported GSAs are based on graphene/polymer composites or stacks, ${ }^{2,11,17}$ whereas the low optical damage threshold of the polymer could severely limit the output power of the laser. Therefore, a feasible, reproducible, and controllable technique is highly desired for the fabrication of GSA.

Plasma-enhanced chemical vapor deposition (PECVD) has emerged as an important method for producing carbon materials such as diamonds, carbon nanotubes and verticallyoriented graphene (VG) nanosheets as well as graphene. ${ }^{18-22}$ The energetic electrons generated by the plasma boost the ionization, excitation and dissociation of hydrocarbon precursors at relatively low temperature. Therefore, graphene can be growth directly on the desired substrates at low temperature by PECVD without metal catalysts. PECVD growth of graphene or

School of Optoelectronic Engineering, Nanjing University of Posts and Telecommunications, Nanjing 210023, China. E-mail: kehanyu@njupt.edu.cn; zxzhang@njupt.edu.cn; Fax: +86-25-85866296

$\dagger$ Electronic supplementary information (ESI) available: The investigations of the growth condition without the rf power and the SEM images of VG. See DOI: $10.1039 / \mathrm{c} 7 \mathrm{ra} 10260 \mathrm{~g}$

$\ddagger$ These authors contributed equally to this work.
VG has been conducted on virtually any substrates ranging from macro-sized planar ${ }^{23-26}$ and cylindrical shapes to millimeter- ${ }^{27}$ micrometer- ${ }^{28}$ and even nanometer-sized ${ }^{29}$ structures. This ability allows the easy fabrication of different graphene-based devices for diverse applications.

Here, we report a one-step fabrication of graphitic nano-layer saturable absorber (GNLSA) on optical fiber-end by PECVD. By changing growth time, desired layer number of graphitic nanolayer (GNL) can be uniformly grown on the optical fiber-end. The fabricated GNLSA was used to mode-lock an erbiumdoped fiber (EDF) laser. The generated optical soliton pulses with the $991 \mathrm{fs}$ duration and $2.7 \mathrm{~nm}$ bandwidth was centered at $1559 \mathrm{~nm}$. The as-prepared GNLSA can operation stably at mode locking state with the maximum pump power of $700 \mathrm{~mW}$. The reported method enables a feasible, reproducible, and controllable technique for the fabrication of GSA.

Single-mode fibers (SMFs) were stripped, wiped up with alcohol, cut by fiber cleaver. Then fiber ends were cleaned by an arc discharge (Fusion Splicer). Fused quartz slides $(1.5 \times$ $1.5 \mathrm{~cm}^{2}, 1 \mathrm{~mm}$ in thickness), used as reference substrates, were cleaned in ultrasonic bathes of acetone and isopropanol, sequentially. Then they were rinsed with deionized water, dried in nitrogen flow.

GNL was produced using a radio-frequency (rf) PECVD system consisting of a tube furnace (OTF-1200X) and a rf generator (VERG-500 RF POWER GENERATOR, $f=13.56 \mathrm{MHz}$ ). The SMF and the fused quartz slide were placed side by side on a homemade graphite holder at the center of the heating area. The reaction chamber was evacuated to $\sim 40$ mTorr, then a mixture of 1 standard cubic centimeter per minute ( $\mathrm{sccm}$ ) of methane $\left(\mathrm{CH}_{4}\right)$ and $9 \mathrm{sccm}$ of hydrogen $\left(\mathrm{H}_{2}\right)$ were introduced into the system when the reactor was heated up to $700{ }^{\circ} \mathrm{C}$. The GNL growth was conducted at $\sim 300$ mTorr, with rf power of $200 \mathrm{~W}$ for $40 \mathrm{~min}$. After the growth, the $\mathrm{CH}_{4}$ flow, $\mathrm{H}_{2}$ flow, and plasma were switched out; the reactor was cooled down in an argon (Ar) flow.

The morphology of GNL grow on fiber-end was observed using a field-emission SEM (Hitachi S4800). Raman 
spectroscopy was carried out with an EZRaman-M Portable Raman System (EZM-785-A2, $\lambda=532 \mathrm{~nm}$ ). The crystal structure of GNL was analyzed by a Bruker D8 polycrystalline X-ray diffraction (D/MAX-2500, $\lambda=1.5406 \AA$ ). Optical transmittance of GNL was tested with a PerkinElmer Lambda 950 machine. The laser performance was characterized by an optical spectrum analyzer (Yokogawa, AQ6370D), a Serial Data Analyzer Oscilloscope (LeCroy, SDA-6000A), a $10 \mathrm{GHz}$ photo-detector (KG-PD-10G), a FROG Scan (Mesaphotonics), and a $30 \mathrm{GHz}$ RF spectrum analyzer (RS-FSV30).

By PECVD, GNL was uniformly grown on the optical fiberend (SMF, $125 \mu \mathrm{m}$ in diameter), as observed under SEM (Fig. 1a). The morphology of the GNL from the core area to the edge area of the fiber-end, as shown in Fig. $1 \mathrm{~b}-\mathrm{d}$, is featured by nano-platelets. No VG was observed under the present condition of growth.

Nanocrystalline nature of the as-grown GNL was revealed by Raman scattering spectrum. Three distinct peaks were observed at $1348 \mathrm{~cm}^{-1}$ (D band), $1584 \mathrm{~cm}^{-1}$ (G band) and $2695 \mathrm{~cm}^{-1}$ (2D band), as shown in Fig. 2a. The $\mathrm{G}$ and $2 \mathrm{D}$ band are the Raman characteristic peaks of $\mathrm{sp}^{2}$-hybridized carbon-carbon bonds in graphene, ${ }^{30,31}$ indicating the formation of graphitized structure. The $\mathrm{D}$ band together with $2 \mathrm{D}^{\prime}$ band $\left(3241 \mathrm{~cm}^{-1}\right)$ is attributed to the structural disorder in the GNL. ${ }^{23}$ The small $2 \mathrm{D}^{\prime}$ band reflects the existence of the $\mathrm{D}^{\prime}$ band that is probably convoluted with the $\mathrm{G}$ band. The intense $\mathrm{D}$ band reveals small crystalline with numerous open edges and defects in the as-grown GNL. The fraction of $\mathrm{sp}^{2}$ carbon in the as-grown GNL is imperfect, which is consistent with reported results. ${ }^{26,32,33}$ Additionally, the X-ray diffraction (XRD) (002) peak at $2 \theta=26.543^{\circ}$ is corresponding to an interlayer spacing $d_{002}=0.335 \mathrm{~nm}$ (Fig. 2b), which is very close to that of pristine few-layer graphene.

The graphene film growth in PECVD is involved with two steps: (a) nucleation: in the early growth stage, precursor (e.g. $\mathrm{CH}_{4}$ ) is dissociated into various reactive radicals in plasma, the carbon-related radicals deposited onto the substrate to form graphene islands; (b) growth: with the continuous growth, higher density of graphene islands form and coalesce into
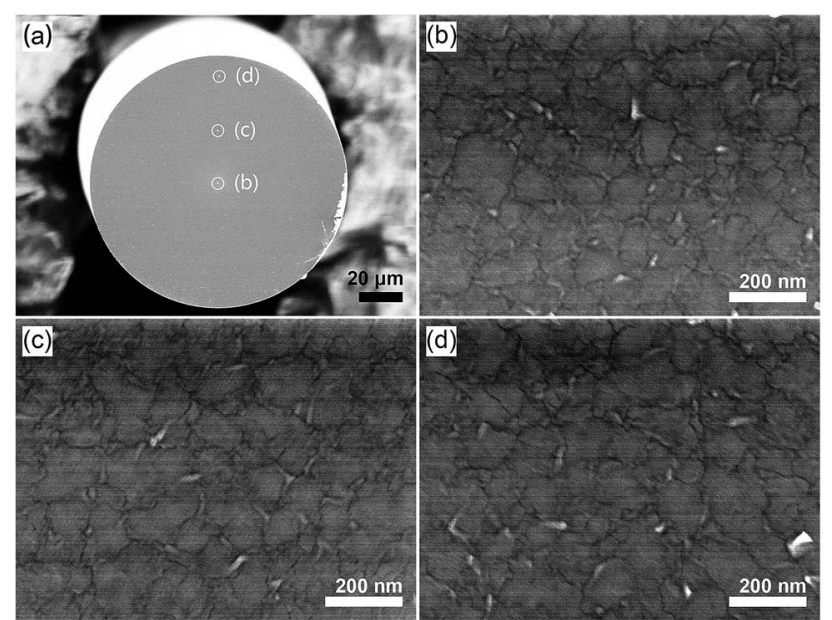

Fig. 1 (a) SEM image of a fiber-end with directly grown GNL. (b)-(d) SEM images taken from the positions of the fiber-end marked in (a).
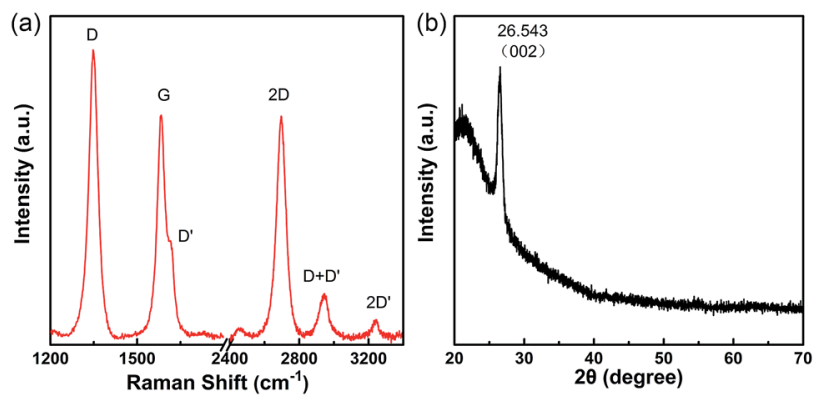

Fig. 2 (a) Raman scattering spectrum and (b) XRD pattern of GNL on quartz substrate.

a continuous and uniform graphene films. ${ }^{18,34}$ With increasing the growth time, the layer number of graphene increased, from few-layer graphene to multilayer graphene, and eventually graphitic layer can be obtained.

The linear transmission spectrum of the as-grown GNL is nearly featureless from 200 to $2400 \mathrm{~nm}$, and with a peak in the ultraviolet region at around $270 \mathrm{~nm}$ (Fig. 3a), which is consistent with graphene made by other techniques. ${ }^{35}$ The number of layers of GNL can be evaluated by a formula ${ }^{36}$

$$
T=\left(1+\frac{1.13 \pi \alpha N}{2}\right)^{-2}
$$

where $\alpha$ is the fine-structure constant $(\approx 1 / 137),{ }^{37} \mathrm{~T}$ is the optical transmittance of GNL at $550 \mathrm{~nm}$, and $N$ is the number of layers. $N$ is calculated to be about 23 corresponding to $T=58.8 \%$ (Fig. 3a). The transmission of GNL is about $66 \%$ at $1550 \mathrm{~nm}$. Note that the calculated number of layers $N$ is an equivalent value for the purpose of estimating the thickness only, in fact the thin film is consist of a larger number of nano-platelets stacking together.

Using a typical twin-detector measurement technology, the nonlinear saturable absorbance property of GNLSA was investigated. The illumination pulse was characterized by a nanotube mode-locked fiber laser delivering $\sim 600$ fs pulse with $23 \mathrm{MHz}$ repetition rate at $1550 \mathrm{~nm}$. As illustrated in Fig. 3b, the asprepared GNLSA exhibits typical feature of saturable absorption, i.e. the light transmission increases with the pulse intensity. The experimental data are fitted through the two-level saturable model
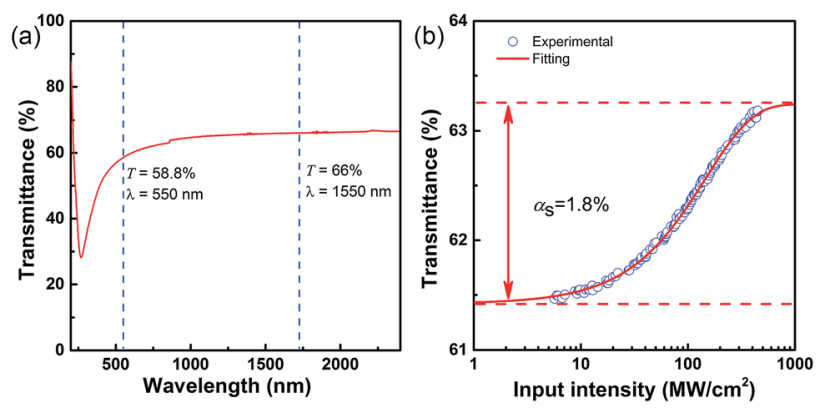

Fig. 3 (a) Transmittance spectrum of an as-grown GNL thin film on quartz substrate. (b) Transmittance of the GNLSA as a function of average pump power. 


$$
\alpha(I)=\frac{\alpha_{\mathrm{s}}}{1+\frac{I}{I_{\mathrm{s}}}}+\alpha_{\mathrm{ns}}
$$

where $\alpha_{\mathrm{s}}$ and $\alpha_{\mathrm{ns}}$ are the saturable and nonsaturable absorption, respectively. $I$ and $I_{\mathrm{s}}$ are the light intensity and the saturation intensity, respectively. The absolute modulation depth is about $1.8 \%$ with $I_{\mathrm{s}}=86 \mathrm{MW} \mathrm{cm}^{-2}$, and $\alpha_{\mathrm{ns}}=36.7 \%$ after fitting the measured data. The nonsaturable absorption is an important parameter to assess the property of an SA, which adverse to high output power of the laser. Mostly, the non-saturable absorption is relevant to the impurities in the SAs. ${ }^{38}$ In this study, the $36.7 \%$ nonsaturable absorption probably be caused by the insertion loss of the bare adapter, scattering of graphene multilayers, ${ }^{1}$ and amorphous carbon in as-grown GNL. The formation of amorphous carbon could be depressed by adjusting the $\mathrm{CH}_{4}: \mathrm{H}_{2}$ ratio and growth temperature. ${ }^{20}$ It worth noting that the modulation depth of a GSA is controlled by the layer number of graphene. For example, multi-layer graphene/PMMA stacks can provide the desired modulation depth. ${ }^{11}$ The direct growth of GNL proposed here greatly simply the fabrication procedure, avoiding the complicate and time consuming growth and post transfer processes.

We constructed an EDF laser to investigate the performance of as-prepared GNLSA. As shown in Fig. 4. The laser system consisted of a wavelength division multiplexer (WDM), a $5 \mathrm{~m}$ EDF with $6 \mathrm{~dB} \mathrm{~m}^{-1}$ absorption at $980 \mathrm{~nm}$, an optical coupler (OC) with output ratio of $10 \%$, a polarization-independent isolator (PI-ISO), a GNL mode-locker, and a polarization controller (PC). The mode-locker is achieved by insert GNLSA into a bare fiber adapter and connect with a fiber adapter by flange (inset of Fig. 4). The dispersion parameters for EDF and SMF are about $-16 \mathrm{ps}(\mathrm{nm} \mathrm{km})^{-1}$ and $17 \mathrm{ps}(\mathrm{nm} \mathrm{km})^{-1}$, respectively. The total cavity length is $\sim 24 \mathrm{~m}$ and the net dispersion is $\sim-0.31 \mathrm{ps}^{2}$.

The threshold pump power for continuous wave lasing was about $50 \mathrm{~mW}$. Fig. 5 summarizes the characteristics of modelocked with the pump power of $200 \mathrm{~mW}$. A mode-locked laser spectrum centering at $1559 \mathrm{~nm}$ is shown in Fig. 5a, with a $3 \mathrm{~dB}$ spectral bandwidth of $2.7 \mathrm{~nm}$. Kelly sidebands at the spectrum shoulders, confirming typical soliton-like pulse formations,

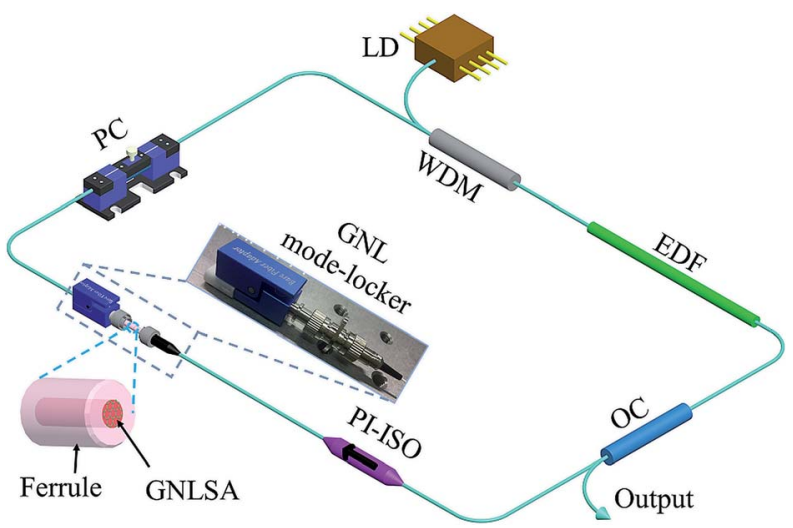

Fig. 4 Schematics of mode-locked fiber laser. Inset: a GNL modelocker.
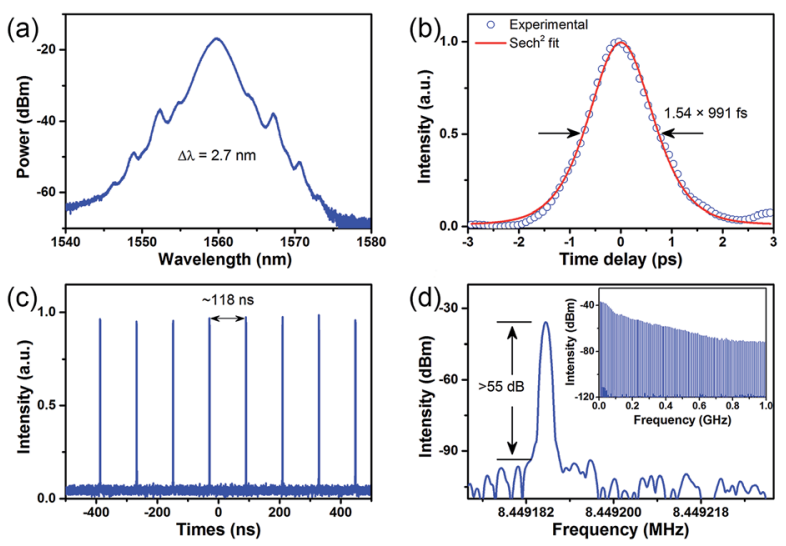

Fig. 5 Mode-locking characteristics. (a) Optical spectrum, (b) autocorrelation trace, (c) pulse train, (d) RF spectrum. Inset: wideband RF spectrum up to $1 \mathrm{GHz}$.

originated from intracavity periodic perturbations. ${ }^{2,39}$ The autocorrelation trace is illustrated in Fig. 5b, and the pulse duration of $991 \mathrm{fs}$ is given by $\operatorname{sech}^{2}$ fitting. The time-bandwidth product of 0.33 is close to the value of the transform-limited sech $^{2}$-shaped pulses. ${ }^{17}$ The repetition period of the output pulses train was $\sim 118 \mathrm{~ns}$, corresponding to the cavity round-trip time (Fig. 5c). The repetition rate of the fundamental cavity frequency of $8.449 \mathrm{MHz}$ together with a radio frequency (RF) spectrum signal-to-noise ratio of $\sim 58 \mathrm{~dB}$ are shown in Fig. $5 \mathrm{~d}$ indicating a laser operation in low-amplitude fluctuations and stability mode-locking regime. ${ }^{\mathbf{4 0 , 4 1}}$ No spectrum modulation was observed over $1 \mathrm{GHz}$ (inset of Fig. 5d), indicating no Q-switching instabilities. ${ }^{\mathbf{4 0 , 4 2}}$ The investigations of the growth condition without the rf power are available in ESI. $\dagger$

An inherent characteristic of the proposed SA is the high optical damage threshold. The mode locking operation worked from the self-started threshold to the maximum available pump power of $700 \mathrm{~mW}$. Fig. 6 shows the average output power and central wavelength as a function of pump power for GNLSA, respectively. The average output power increases linearly as the

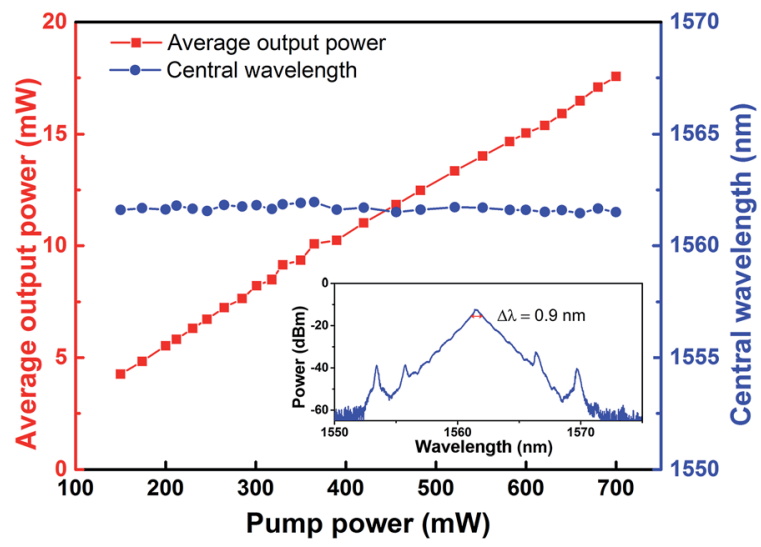

Fig. 6 Average output power and central wavelength versus pump power at the mode-locking operation state. Inset: spectrum at pump power of $700 \mathrm{~mW}$. 
pump power increasing; the central wavelength almost keeps constant, and none output power drop is observed, indicating a robust GNLSA resistant to thermal damage. The inset in Fig. 6 shows the optical spectrum of stable soliton mode locking by increasing the pump power to $700 \mathrm{~mW}$.

It is worthwhile noting that the VG can be grown on optical fiber-ends with a fast growth, e.g. PECVD at higher temperature. But no mode-locked laser was obtained using a VG coated optical fiber. Calculated with eqn (1), the VG is equivalent to a 32-layered GNL. The insertion loss of VG is perhaps too high to output mode-locked laser. The SEM images of VG grown on optical fiber-end are available in ESI. $\dagger$

In conclusion, we report a one-step fabrication of GNLSA on optical fiber-end for passively mode-locked laser by PECVD. Without catalyst, nanocrystalline GNL with controllable number of layers was uniformly grown on the optical fiber-end, exhibiting a modulation depth of $1.8 \%$ with a 23 -layered GNLSA. A stable mode-locked fiber laser was achieved using a 23-layered GNLSA with spectrum centered at $1559 \mathrm{~nm}, 3 \mathrm{~dB}$ bandwidth of $2.7 \mathrm{~nm}$, soliton pulses of $991 \mathrm{fs}$, and repetition rate of $8.449 \mathrm{MHz}$. The as-grown GNLSA can bear pump power up to $700 \mathrm{~mW}$, the average output power reached $17.6 \mathrm{~mW}$. This feasible, clean and controllable fabrication approach may greatly facilitate the development of the fabrication of graphene based devices.

\section{Conflicts of interest}

There are no conflicts to declare.

\section{Acknowledgements}

This work was financially supported by the National Natural Science Foundation of China No. 61504064, the Natural Science Foundation of Jiangsu Province, China (No. BK20150847 and No. BK20161521), and NUPTSF Grant no. NY214137 and no. NY214002.

\section{References}

1 Q. Bao, H. Zhang, Y. Wang, Z. Ni, Y. Yan, Z. X. Shen, K. P. Loh and D. Y. Tang, Adv. Funct. Mater., 2009, 19, 3077-3083.

2 Z. Sun, T. Hasan, F. Torrisi, D. Popa, G. Privitera, F. Wang, F. Bonaccorso, D. M. Basko and A. C. Ferrari, ACS Nano, 2010, 4, 803-810.

3 Z. Sun, T. Hasan and A. C. Ferrari, Phys. E, 2012, 44, 10821091.

4 A. Martinez and Z. Sun, Nat. Photonics, 2013, 7, 842-845.

5 Y. M. Chang, H. Kim, J. H. Lee and Y.-W. Song, Appl. Phys. Lett., 2010, 97, 211102.

6 A. Martinez, K. Fuse and S. Yamashita, Appl. Phys. Lett., 2011, 99, 121107.

7 F. Bonaccorso and Z. Sun, Opt. Mater. Express, 2014, 4, 63-78. 8 D. Popa, Z. Sun, F. Torrisi, T. Hasan, F. Wang and A. C. Ferrari, Appl. Phys. Lett., 2010, 97, 831-833.
9 Q. Bao, H. Zhang, J. X. Yang, S. Wang, D. Y. Tang, R. Jose, S. Ramakrishna, C. T. Lim and K. P. Loh, Adv. Funct. Mater., 2010, 20, 782-791.

10 H. Zhang, D. Y. Tang, R. J. Knize, L. Zhao, Q. Bao and K. P. Loh, Appl. Phys. Lett., 2010, 96, 111112.

11 G. Sobon, J. Sotor, I. Pasternak, A. Krajewska, W. Strupinski and K. M. Abramski, Opt. Mater. Express, 2015, 5, 2884-2894.

12 H. Zhang, D. Y. Tang, L. Zhao, Q. Bao and K. P. Loh, Opt. Express, 2009, 17, 17630-17635.

13 G. Zhu, X. Zhu, F. Wang, S. Xu, Y. Li, X. Guo, K. Balakrishnan, R. A. Norwood and N. Peyghambarian, IEEE Photonics Technol. Lett., 2016, 28, 7-10.

14 Y.-W. Song, S.-Y. Jang, W.-S. Han and M.-K. Bae, Appl. Phys. Lett., 2010, 96, 051122.

15 H. Zhang, Q. Bao, D. Y. Tang, L. Zhao and K. P. Loh, Appl. Phys. Lett., 2009, 95, 141103.

16 F. Bonaccorso, A. Lombardo, T. Hasan, Z. Sun, L. Colombo and A. C. Ferrari, Mater. Today, 2012, 15, 564-589.

17 Z. Sun, D. Popa, T. Hasan, F. Torrisi, F. Wang, E. J. R. Kelleher, J. C. Travers, V. Nicolosi and A. C. Ferrari, Nano Res., 2010, 3, 653-660.

18 M. Li, D. Liu, D. Wei, X. Song, D. Wei and A. T. S. Wee, Adv. Sci., 2016, 3, 1600003.

19 Z. Bo, S. Mao, Z. J. Han, K. Cen, J. Chen and K. Ostrikov, Chem. Soc. Rev., 2015, 44, 2108-2121.

20 Z. Bo, Y. Yang, J. Chen, K. Yu, J. Yan and K. Cen, Nanoscale, 2013, 5, 5180-5204.

21 K. Ostrikov, Rev. Mod. Phys., 2005, 77, 489-511.

22 K. Ostrikov and A. B. Murphy, J. Phys. D: Appl. Phys., 2007, 40, 2223-2241.

23 K. Yu, Z. Bo, G. Lu, S. Mao, S. Cui, Y. Zhu, X. Chen, R. S. Ruoff and J. Chen, Nanoscale Res. Lett., 2011, 6, 202-210.

24 H. Bi, S. Sun, F. Huang, X. Xie and M. Jiang, J. Mater. Chem., 2012, 22, 411-416.

25 Y. S. Kim, K. Joo, S.-K. Jerng, J. H. Lee, D. Moon, J. Kim, E. Yoon and S.-H. Chun, ACS Nano, 2014, 8, 2230-2236.

26 L. Zhang, Z. Shi, Y. Wang, R. Yang, D. Shi and G. Zhang, Nano Res., 2011, 4, 315-321.

27 Z. Bo, K. Yu, G. Lu, S. Cui, S. Mao and J. Chen, Energy Environ. Sci., 2011, 4, 2525-2528.

28 H.-C. Chang, H.-Y. Chang, W.-J. Su, K.-Y. Lee and W.-C. Shih, Appl. Surf. Sci., 2012, 258, 8599-8602.

29 K. Yu, G. Lu, Z. Bo, S. Mao and J. Chen, J. Phys. Chem. Lett., 2011, 2, 1556-1562.

30 A. C. Ferrari, J. C. Meyer, V. Scardaci, C. Casiraghi, M. Lazzeri, F. Mauri, S. Piscanec, D. Jiang, K. S. Novoselov, S. Roth and A. K. Geim, Phys. Rev. Lett., 2006, 97, 187401.

31 D. Graf, F. Molitor, K. Ensslin, C. Stampfer, A. Jungen, C. Hierold and L. Wirtz, Nano Lett., 2006, 7, 238-242.

32 T. E. Benavidez, R. Martinez-Duarte and C. D. Garcia, Anal. Methods, 2016, 8, 4163-4176.

33 X. Chen, B. Wu and Y. Liu, Chem. Soc. Rev., 2016, 45, 20572074.

34 W. Yang, C. He, L. Zhang, Y. Wang, Z. Shi, M. Cheng, G. Xie, D. Wang, R. Yang, D. Shi and G. Zhang, Small, 2012, 8, 14291435. 
35 F. Bonaccorso, Z. Sun, T. Hasan and A. C. Ferrari, Nat. Photonics, 2010, 4, 611-622.

36 S. E. Zhu, S. Yuan and G. C. A. M. Janssen, Europhys. Lett., 2014, 108, 17007-17010.

37 R. R. Nair, P. Blake, A. N. Grigorenko, K. S. Novoselov, T. J. Booth, T. Stauber, N. M. R. Peres and A. K. Geim, Science, 2008, 320, 1308.

38 L. Li, S. Jiang, Y. Wang, X. Wang, L. Duan, D. Mao, Z. Li, B. Man and J. Si, Opt. Express, 2015, 23, 28698-28706.
39 M. L. Dennis and I. N. Duling, IEEE J. Quantum Electron., 1994, 30, 1469-1477.

40 F. Wang, A. G. Rozhin, V. Scardaci, Z. Sun, F. Hennrich, I. H. White, W. I. Milne and A. C. Ferrari, Nat. Nanotechnol., 2008, 3, 738-742.

41 D. Von der Linde, Appl. Phys. B, 1986, 39, 201-217.

42 U. Keller, K. J. Weingarten, F. X. Kärtner, D. Kopf, B. Braun, I. D. Jung, R. Fluck, C. Hönninger, N. Matuschek and J. A. D. Au, IEEE J. Sel. Top. Quantum Electron., 1996, 2, 435-453. 